\section{Reduced structural connectivity in ventral visual cortex in congenital prosopagnosia}

Cibu Thomas ${ }^{1,2}$, Galia Avidan ${ }^{3}$, Kate Humphreys ${ }^{4}$, Kwan-jin Jung ${ }^{5}$ Fuqiang $\mathrm{Gao}^{6}$ \& Marlene Behrmann ${ }^{1,2}$

\begin{abstract}
Using diffusion tensor imaging and tractography, we found that a disruption in structural connectivity in ventral occipitotemporal cortex may be the neurobiological basis for the lifelong impairment in face recognition that is experienced by individuals who suffer from congenital prosopagnosia. Our findings suggest that white-matter fibers in ventral occipitotemporal cortex support the integrated function of a distributed cortical network that subserves normal face processing.
\end{abstract}

Prosopagnosia is characterized by a profound impairment in recognizing faces, despite normal sensory vision and intelligence. In contrast with acquired prosopagnosia, which typically results from a neurological insult to the ventral visual cortex in premorbidly normal individuals, individuals with congenital prosopagnosia have impaired face recognition, apparently from birth, and in the absence of any obvious insult to the brain ${ }^{1}$. Therefore, we explored the neurobiological substrate of congenital prosopagnosia.

Given that face processing is mediated by a widely distributed network, comprising 'core' regions in the ventral occipito-temporal cortex and 'extended' regions in anterior temporal and frontal cortices ${ }^{2}$, it is conceivable that congenital prosopagnosia might be attributable to abnormal functioning of the core regions. However, studies have failed to definitively implicate the core regions as the source of the impairment; face-selective electrophysiological markers, derived from evoked response potentials ${ }^{3}$ or magnetoencephalography ${ }^{4}$, have been shown to be normal in many congenital prosopagnosic individuals, albeit not in all (perhaps a function of the heterogeneity in the population), and several functional magnetic resonance imaging studies have reported a normal cortical response in individuals with congenital prosopagnosia in the magnitude, site and adaptation profile of the BOLD activation $^{5-7}$. Therefore, a plausible alternative hypothesis is that congenital prosopagnosia emerges from a disruption in the structural connectivity between the nodes of the distributed face-processing network. This hypothesis is also consistent with the fact that there is a reduction in the volume of the anterior fusiform gyrus in congenital prosopagnosia ${ }^{8}$, but whether this reduction is attributable to decreases in gray and/or white matter is not yet known.
To test this hypothesis using diffusion tensor imaging (DTI), we evaluated the structural integrity of the white-matter tracts in ventral occipito-temporal cortex (VOTC) in congenital prosopagnosic individuals ( $n=6$, aged $57.7 \pm 15.2$ years), all of whom evinced normal BOLD activation in the core face regions ${ }^{6}$ relative to matched controls ( $n=17$, aged $55.5 \pm 18.5$ years) (Supplementary Methods online). We used a systematic protocol ${ }^{9}$ to extract and quantify the macro- and microstructural properties of the inferior longitudinal fasciculus (ILF) and the inferior fronto-occipito fasciculus (IFOF) ${ }^{10}$, the two major tracts that project through the core fusiform region to the anterior temporal and frontal cortices, respectively (Supplementary Fig. 1 online). To determine the specificity of any tract perturbations, we also examined two callosal tracts, the forceps major, which links the bilateral occipital lobes through the splenium, and the forceps minor, which links the bilateral frontal lobes through the rostrum and genu. In a converging, assumption-free analysis, we sought group-wise differences in fractional anisotropy, a measure of microstructural integrity of white matter, at a voxel level across the whole brain (Supplementary Methods). Finally, we correlated the face-recognition performance of the individuals with congenital prosopagnosia with the connectivitydependent measures to assess the functional relevance of alterations in structural connectivity.

Relative to the controls, the congenital prosopagnosia group showed a marked reduction in the structural integrity of the ILF and IFOF bilaterally (Fig. 1) across all measures (Supplementary Fig. 2 online for all plots and Supplementary Table 1 online for statistics). The congenital prosopagnosia group also showed a substantial reduction in the volume of the forceps major, but this was not consistent across all dependent measures. There were no notable group differences in the integrity of the forceps minor (Fig. 2a), limiting the perturbation to the white-matter tracts projecting through the VOTC. The whole brain analyses revealed a significant reduction in fractional anisotropy, along the trajectory of the ILF and of the IFOF, in the white matter adjacent to the right and left fusiform gyrus, right anterior temporal stem and the left external capsule in the congenital prosopagnosia group $(P<0.007$, uncorrected; Fig. 1c,d). This offers converging evidence for a reduction in microstructural integrity of the white-matter tracts along the VOTC that link the core regions with the extended regions of the face network.

To examine the functional consequences of these structural differences, we tested the face-recognition abilities of both the congenital prosopagnosia group and a subgroup of the controls (see Supplementary Methods). As expected, the congenital prosopagnosia subjects performed more poorly than their control counterparts $(t=-6.28, P<0.001)$. We then carried out a stepwise regression analysis with the connectivity and behavioral measures; because of the limited size of the congenital prosopagnosia group, and as our goal was

${ }^{1}$ Department of Psychology, Carnegie Mellon University, 5000 Forbes Avenue, Pittsburgh, Pennsylvania 15213, USA. ${ }^{2}$ Center for the Neural Basis of Cognition, 4400 Fifth Avenue, Pittsburgh, Pennsylvania 15213, USA. ${ }^{3}$ Department of Behavioral Sciences, Ben-Gurion University of the Negev, P.0.B. 653, Beer-Sheva, 84105, Israel. ${ }^{4}$ Institute of Psychiatry, Kings' College London, De Crespigny Park, London, SE5 8AF, UK. ${ }^{5}$ Brain Imaging Research Center, 3025 East Carson Street, University of Pittsburgh and Carnegie Mellon University, Pittsburgh, Pennsylvania 15203, USA. ${ }^{6}$ Sunnybrook Health Sciences Center, 2075 Bayview Avenue, University of Toronto, Toronto, Ontario M4N 3M5, Canada. Correspondence should be addressed to C.T. (cibut@nmr.mgh.harvard.edu).

Received 1 May; accepted 14 October; published online 23 November 2008; doi:10.1038/nn.2224 
a Congenital prosopagnosia individuals ILF
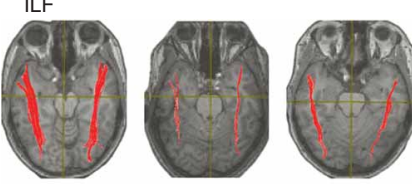

IFOF

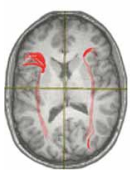

F, 33

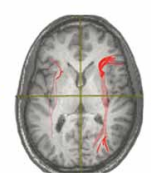

M, 49

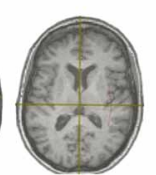

F, 63
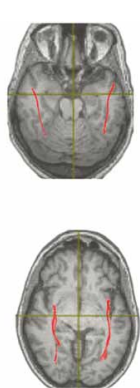

F, 64
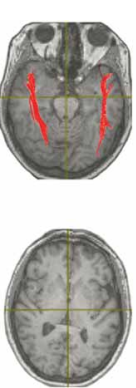

$\mathrm{F}, 67$
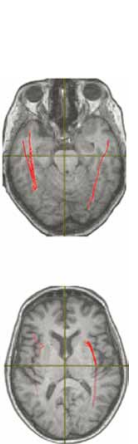

F, 72 b Matched control individuals ILF
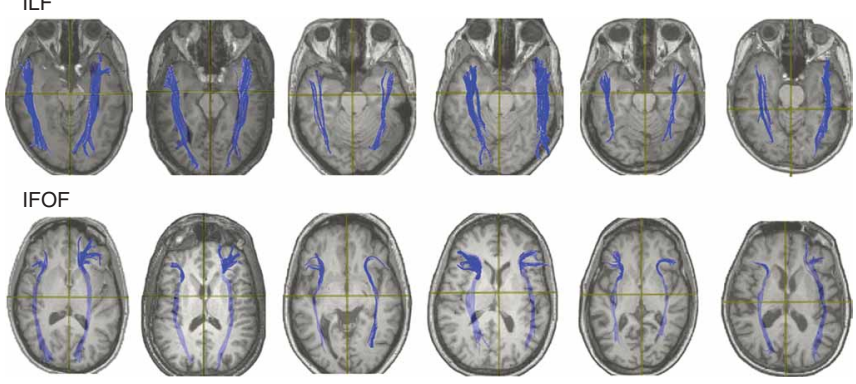

F, 36

M, 50

$\mathrm{F}, 60$

$\mathrm{F}, 71$

F, 80

d Relative trajectories of ILF and IFOF in ventral temporal cortex
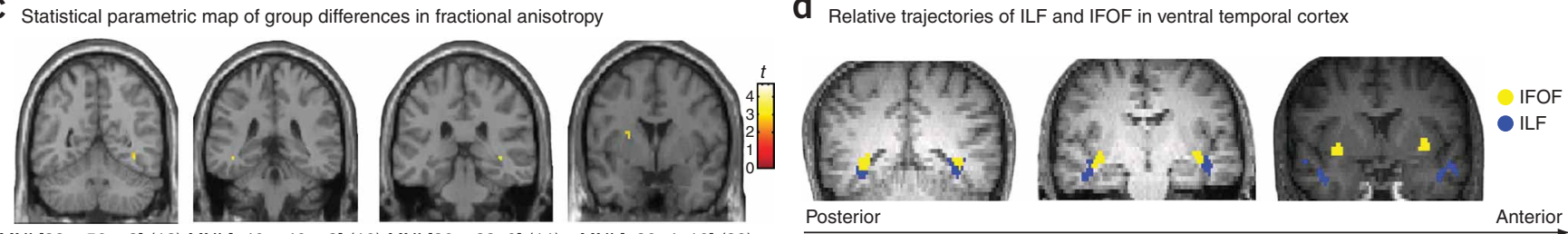

MNI $[38,-56,-8](13)$ MNI $[-40,-40,-8](10)$ MNI $[36,-32,0](11) \quad$ MNI $[-26,4,16](29)$

Figure 1 Tractography reveals a profound alteration in the structural integrity of the ILF and the IFOF in individuals with congenital prosopagnosia compared with their matched controls. Further analyses confirmed a reduction in the microstructural integrity of white matter along the trajectory of the ILF and IFOF in both hemispheres. (a,b) Axial slices showing the bilateral ILF and the IFOF in individuals with congenital prosopagnosia and their matched controls. The gender and age of each individual with congenital prosopagnosia and their matched control is indicated below each slice. (c,d) The whole-brain voxel-wise fractional anisotropy analysis revealed a reduction in fractional anisotropy (c) in four regions along the trajectory (d) of the ILF (blue) and IFOF (yellow) in the congenital prosopagnosia group. Montreal Neurological Institute (MNI) coordinates and the number of voxels are provided below each image. All images are displayed in neurological convention.

to elucidate the relationship between group-wide differences in structural connectivity and behavior, we included both the congenital prosopagnosia and control data. The analysis revealed a significant negative correlation between errors in face recognition and a reduction in mean fractional anisotropy (slope $=-0.635, t=-2.73, P<0.02$, $r^{2}=0.40$ ) and percentage of voxels (slope $=-0.726, t=-3.5$, $\left.P<0.005, r^{2}=0.53\right)$ in the right ILF. Furthermore, a reduction in the percentage of fibers in the right IFOF correlated with poor face recognition (slope $=-0.74, t=-3.7$, $P<0.004, r^{2}=0.55$; Fig. 2b-d).

In summary, our study reveals for the first time to the best of our knowledge, that the characteristic behavioral profile of congenital prosopagnosia may be ascribed to a disruption in structural connectivity in the ILF and, perhaps to a lesser extent, the IFOF. The finding that only the reduction in tract integrity in the right hemisphere and behavioral

Figure 2 The relation between alterations in structural connectivity in ventral occipito-temporal cortex and poor face-recognition ability. (a) The congenital prosopagnosia group showed a reduction in the microstructural integrity of the bilateral ILF and IFOF, but not in the two callosal tracts. $\mathrm{LH}$, left hemisphere; $\mathrm{RH}$, right hemisphere. (b) A reduction in the percentage of fibers in the right IFOF was associated with an increase in errors in face recognition. (c,d) Similarly, a reduction in the mean fractional anisotropy (c) and percentage of voxels (d) in the right ILF were also associated with an increase in errors in face recognition. Error bars indicate s.e.m. accuracy were correlated dovetails with the prevailing notion that the right hemisphere is more prominent in face processing ${ }^{2}$. These results are also compatible with the finding that white-matter lesions in the ILF as a result of multiple sclerosis may induce symptoms of prosopagnosia and object agnosia ${ }^{11}$. Given that feedback from frontal cortex is important in higher-level visual processing ${ }^{12-14}$, it is conceivable that a disruption in the IFOF, which links frontal cortex to VOTC, also contributes to deficits in face processing. Closer scrutiny of the data
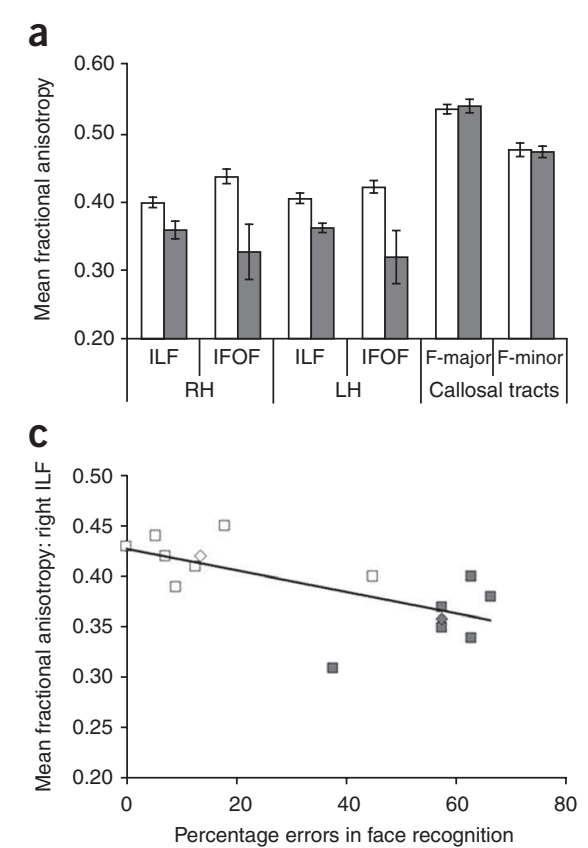

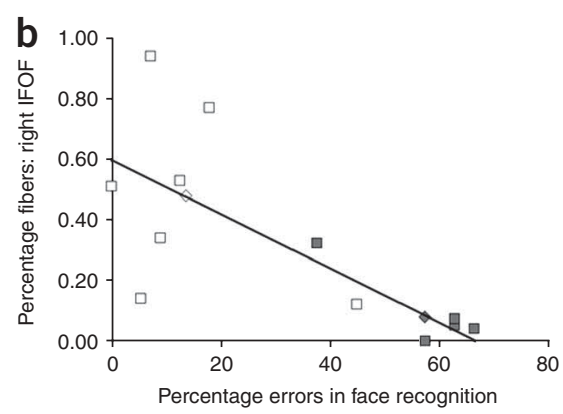

d

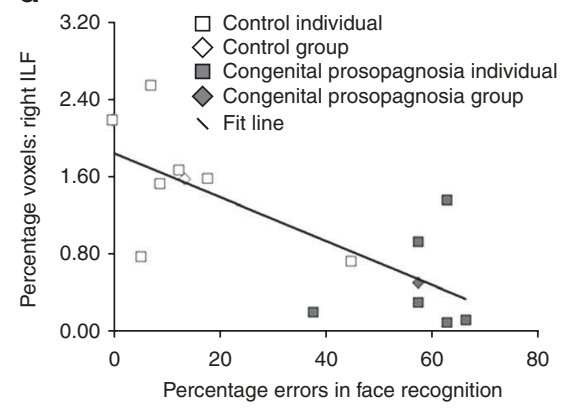


indicated that there was some variability in the exact pattern of the ILF and IFOF perturbation across the different congenital prosopagnosic individuals in the sample. Future studies should examine whether there are subpatterns of congenital prosopagnosia; assessing the relation between the tract measures and measures of face discrimination and/or configural processing may help to elucidate the functional implications of certain patterns of disruption and the concomitant difficulty in face processing.

In conclusion, we have uncovered a potential neurobiological substrate for congenital prosopagnosia. Confirming this in a larger sample with more sophisticated diffusion imaging techniques will shed further light on the structure of these cortical tracts and their role in behavior. This is important, as DTI-based tractography does not involve tracing fibers in the same way that axonal tracers are used to establish connectivity maps, which means that absolute values yielded by tractography, such as density and volume of fibers, may not be anatomically veridical. Indeed, the sparsity of VOTC fibers in congenital prosopagnosia does not necessarily indicate that the fibers are absent entirely. Rather, it suggests that, relative to the controls, the structural integrity of specific white-matter tracts is so compromised as to preclude the extraction of the tracts.

Finally, the discovery of a reduction in VOTC fiber tracts in congenital prosopagnosia opens up the promise of exposing relationships between the neurodevelopment of white-matter connectivity, behavior and genetics. There is a clear familial component in congenital prosopagnosia and recent studies point to a genetic mutation whose mode of inheritance is autosomal dominant ${ }^{15}$. One possibility, therefore, is that the abnormal expression of a gene during a critical period in brain development may disrupt normal maturation of the whitematter tracts in the VOTC. Further investigation of the neurobiological basis of congenital prosopagnosia will not only shed light on the mechanisms underlying this disorder and, more generally, face processing, but will also enhance our understanding of the genetic and maturational bases of other related neurodevelopmental disorders.
Note: Supplementary information is available on the Nature Neuroscience website.

\section{ACKNOWLEDGMENTS}

We are grateful to S. Mori and H. Jiang of Johns Hopkins University who provided advice and guidance at various stages of this project regarding the development of the DTI protocol and method of analysis. We thank S. Kurdilla and D. Viszlay of the Brain Imaging Research Center for their help in the acquisition of the imaging data and B. Frye and T. Keller for their help with the computation and analyses of fractional anisotropy maps. This study was funded by grants from the US National Institute of Mental Health (MH54246) to M.B. and by awards from the National Alliance for Autism Research (Autism Speaks) to C.T. and K.H. and from the Cure Autism Now foundation to K.H.

\section{AUTHOR CONTRIBUTIONS}

C.T conducted the experiment, undertook the majority of the data analysis and wrote the manuscript. G.A. and K.H. assisted with characterizing the subjects and collecting the behavioral data. K.J. and F.G. contributed to data analysis. M.B. contributed to writing the manuscript and supervised the project.

Published online at http://www.nature.com/natureneuroscience/ Reprints and permissions information is available online at http://npg.nature.com/ reprintsandpermissions/

1. Behrmann, M. \& Avidan, G. Trends Cogn. Sci. 9, 180-187 (2005).

2. Ishai, A., Schmidt, C.F. \& Boesiger, P. Brain Res. Bull. 67, 87-93 (2005).

3. Minnebusch, D.A., Suchan, B., Ramon, M. \& Daum, I. Eur. J. Neurosci. 25, 2234-2247 (2007).

4. Harris, A.M., Duchaine, B.C. \& Nakayama, K. Neuropsychologia 43, 2125-2136 (2005).

5. Hadjikhani, N. \& De Gelder, B. Hum. Brain Mapp. 16, 176-182 (2002).

6. Avidan, G., Hasson, U., Malach, R. \& Behrmann, M. J. Cogn. Neurosci. 17, 1150-1167 (2005).

7. Hasson, U., Avidan, G., Deouell, L.Y., Bentin, S. \& Malach, R. J. Cogn. Neurosci. 15, 419-431 (2003)

8. Behrmann, M., Avidan, G., Gao, F. \& Black, S. Cereb. Cortex 17, 2354-2363 (2007).

9. Thomas, C. et al. J. Cogn. Neurosci. 20, 268-284 (2008).

10. Mori, S. et al. Magn. Reson. Med. 47, 215-223 (2002).

11. Yamasaki, T. et al. J. Neurol. Sci. 221, 53-60 (2004).

12. Tsao, D.Y., Schweers, N., Moeller, S. \& Freiwald, W.A. Nat. Neurosci. 11, 877-879 (2008).

13. Summerfield, C. et al. Science 314, 1311-1314 (2006).

14. Bar, M. et al. Proc. Natl. Acad. Sci. USA 103, 449-454 (2006).

15. Grueter, M. et al. Cortex 43, 734-749 (2007). 